\title{
NORMAL SPACES WITH PRESCRIBED STONE-ČECH REMAINDERS
}

\author{
JACK R. PORTER AND R. GRANT WOODS
}

(Communicated by Dennis Burke)

\begin{abstract}
It is shown that if $X$ is a locally compact Hausdorff space, then there is a normal space $Y$ such that $\beta Y \backslash Y \cong X$. Examples are given of a countable nonsequential space, and a sequential nonlocally compact space, each of which is the Stone-Čech remainder of a normal space. A method of constructing normal almost compact spaces is presented.
\end{abstract}

\section{INTRODUCTION}

It is a well-known consequence of Glicksberg's theorem (see, for example, [PW, 4AG(7)] ) concerning the Stone-Čech compactification of a pseudocompact product that given any Tychonoff space $X$, there exists a Tychonoff space $Y$ such that $\beta Y \backslash Y=X$ (equations such as this will be used to abbreviate the more precise statement " $\beta Y \backslash Y$ is homeomorphic to $X$ "). One can select the space $Y$ to be the subspace $P \backslash(\{\kappa\} \times X)$ of the product $P=[0, \kappa] \times \beta X$, where $\kappa$ is an ordinal of uncountable cofinality; then $P=\beta Y$. (Here $[0, \kappa)$ (resp. $[0, \kappa]$ ) denotes the set of all ordinals less than (resp. less than or equal to) the ordinal $\kappa$, equipped with the order topology.) It is clear that if $X$ is not locally compact then $Y$ will not be normal, for if $x \in X \cap \operatorname{cl}_{\beta X}(\beta X \backslash X)$, then the point $(\kappa, x)$ is in the $P$-closure of the disjoint closed sets $[0, \kappa) \times\{\kappa\}$ and $\{\kappa\} \times(\beta X \backslash X)$ of $Y$. Hence if we are given a nonlocally compact space $X$ and seek a normal space $Y$ such that $X=\beta Y \backslash Y$, we will need a new technique.

Given the above result, it is natural to ask whether any Tychonoff space $X$ is homeomorphic to $\beta T \backslash T$ for some normal space $T$. Perhaps surprisingly, the answer is "no." In [FL] Fleissner and Levy study normal spaces all of whose regular continuous images are normal, and in the course of their investigations obtain constraints on those spaces $X$ that can be $\beta Y \backslash Y$ for some normal space $Y$. Specifically, they prove that if $Y$ is normal and $\beta Y \backslash Y$ is first countable, then $\beta Y \backslash Y$ is locally compact (see [FL, Proposition 4]). Thus, in contrast to

Received by the editors May 31, 1990 and, in revised form, September 17, 1990; presented to the AMS, January 1990, in Louisville, Kentucky.

1980 Mathematics Subject Classification (1985 Revision). Primary 54D40; Secondary 54D55, 54D35, 54D45, 54D15.

Key words and phrases. Stone-Čech compactification, normal, almost compact, sequential, locally compact.

Research of the second-named author was partially supported by Grant A7592 from the Natural Sciences and Engineering Research Council of Canada. 
the situation described in the preceding paragraph, not every (Tychonoff) space $X$ is homeomorphic to $\beta Y \backslash Y$ for some normal space $Y$. (The rationals will be such an $X$, for example.) This raises the question of characterizing those Tychonoff spaces that are homeomorphic to $\beta Y \backslash Y$ for some normal space $Y$. In particular, in Question 1 of [FL], Fleissner and Levy ask whether, if $Y$ is normal, $\beta Y \backslash Y$ can be countable but not sequential, or sequential but not locally compact.

In this paper we show that if $X$ is locally compact, then there is a normal space $Y$ such that $X=\beta Y \backslash Y$. We also show that the answer to each of the questions of Fleissner and Levy quoted above is "yes," although the assumption of the existence of a scale is necessary for our argument in the second case. In the process we develp a new general technique for constructing examples of normal spaces with interesting Stone-Čech compactifications. We conclude the paper with a result concerning normal almost compact spaces that is unrelated to the previous results.

All hypothesized topological spaces in this paper are assumed to be Tychonoff; thus "space" means "completely regular Hausdorff topological space." The reader is referred to [PW] and [GJ] for unexplained notation and terminology. We will make use of the following result, which is Theorem 2.1 of [M].

Lemma 1. Suppose that $X$ is locally compact and $K$ is Hausdorff. Then there exists a compactification $\alpha X$ of $X$ such that $\alpha X \backslash X=K$ if and only if $K$ is a continuous image of $\beta X \backslash X$.

We will also use the following slight generalization of one direction of a theorem of Nogura [N], which essentially appears (with proof) as 3.19 of [P]. Recall that the tightness $t(X)$ of a space $X$ is the smallest cardinal number $\kappa$ such that for every point $x \in X$ and $S \subseteq X$, if $x \in \operatorname{cl}(S)$ then $x \in \operatorname{cl}(T)$ for some $T \subseteq S$ with $|T| \leq \kappa$ (see [H]). As usual, $\operatorname{cf}(\kappa)$ is the cofinality of the cardinal $\kappa$.

Theorem 1. If $\kappa$ is a cardinal number and $C$ is a compact space for which $t(C)<\operatorname{cf}(\kappa)$, then the product space $[0, \kappa) \times C$ is normal.

We can now present our first result.

Theorem 2. Let $X$ be a locally compact space. Then there exists a normal space $Y$ such that $\beta Y \backslash Y=X$.

Proof. Assume without loss of generality that $X$ is infinite. Let $\kappa=|\beta X|^{+}$, $P=[0, \kappa] \times \beta X$, and $Y=P \backslash(\{\kappa\} \times X)$. Thus $[0, \kappa) \times \beta X \subseteq Y \subseteq P=$ $\beta([0, \kappa) \times \beta X)$; the latter equality essentially follows from Glicksberg's theorem (see $[\mathrm{PW}, 4 \mathrm{AG}(8)]$, for example). Thus $\beta Y=P$ (see $[\mathrm{PW}, 4.5(\mathrm{p})(3)])$ and so $\beta Y \backslash Y=\{\kappa\} \times X \cong X$. Clearly, $t(\beta X)<\operatorname{cf}(\kappa)$, so by Theorem $1, H=$ $[0, \kappa) \times \beta X$ is normal. But $Y=H \cup([0, \kappa] \times(\beta X \backslash X))$ and $\beta X \backslash X$ is compact as $X$ is locally compact, so by [GJ, $3 \mathrm{D}(5)], Y$ is normal.

\section{THE BASIC CONSTRUCTION}

Let $S$ be a locally compact noncompact space whose pseudocompact closed subspaces are compact. (This last condition will be fulfilled if $S$ is realcompact 
(see [GJ, 8.10 and $8 \mathrm{~A}(5)]$ ), or metacompact (see [PW, 6N]).) Suppose that $S$ has a compactification $\alpha S$ for which $\alpha S \backslash S=[0, \lambda]$, where $\lambda$ is a cardinal such that $\operatorname{cf}(\lambda)>t(S)$. In Theorem 4 below we will show that there exists a normal space $Y$ for which $\beta Y \backslash Y=S \cup\{\lambda\}$. We will use this construction in $\S 3$ to answer the two questions of Fleissner and Levy posed in $\S 1$.

A central part of our construction of $Y$ will be a generalization of a construction due to Vermeer [V]. The special case of Theorem 3 below in which $\lambda=\omega_{1}$ appears in 3.4 of [V]; the proof of Theorem 3 is virtually identical to Vermeer's proof of 3.4, and hence is not included. (See also [PW, 6W].)

Theorem 3. Let $\lambda$ be a cardinal of uncountable cofinality and let $P=[0, \lambda] \times$ $[0, \lambda]$. If $\alpha<\lambda$, let $E_{\alpha}=\{(\lambda, \alpha),(\alpha, \alpha),(\alpha, \lambda)\}$ and $E=\bigcup\left\{E_{\alpha}: \alpha<\lambda\right\}$. Let $T=\left\{E_{\alpha}: \alpha<\lambda\right\} \cup\{\{p\}: p \in P \backslash E\}$ and let $f: P \rightarrow T$ map each point of $P$ to the unique member of $T$ to which it belongs. Give $T$ the quotient topology induced by $f$. Then $T$ is a compact Hausdorff space, $T \backslash\{\{(\lambda, \lambda)\}\}=Z$ is normal, and $\beta Z=T$.

Theorem 4. Let $S$ be a locally compact noncompact space whose pseudocompact closed subsets are compact. Let $S$ have a compactification $\alpha S$ for which $\alpha S \backslash S=$ $[0, \lambda]$, where $\lambda$ is a cardinal for which $\operatorname{cf}(\lambda)>t(S)$. Then there exist a normal space $Y$ for which $\beta Y \backslash Y=S \cup\{\lambda\}$ (viewed as a subspace of $\alpha S$ ).

Proof. Let $K=[0, \lambda] \times \alpha S$, and let $T$ and $Z$ be as in Theorem 3. Then $K$ is a compactification of the locally compact space $L=[0, \lambda] \times S$, and $K \backslash L=$ $[0, \lambda] \times[0, \lambda]=P$. Define a map $F: K \rightarrow L \dot{U} T=H$ as follows: $F(x)=x$ if $x \in L$, and $F \mid P=f$ (as described in Theorem 3). Give $H$ the quotient topology induced by $F$. By Lemma $1, H$ is a Hausdorff compactification of $L$ and $F$ is a continuous surjection; note that the subspace topologies inherited by $L$ and $T$ from $H$ are the same as the orginal topologies on $L$ and $T$. Observe that $F^{\leftarrow}(F(x))=\{x\}$ if $x \in\{\lambda\} \times(S \cup\{\lambda\})$, so the subspace $(\{\lambda\} \times S) \cup$ $\{\{(\lambda, \lambda)\}\}$, henceforth denoted as $E$, of $H$ is homeomorphic to the subspace $S \cup\{\lambda\}$ of $\alpha S$.

Denote the point $(\lambda, \lambda)$ of $H$ by $a$. Let $Y=H \backslash E$; thus $Y=([0, \lambda) \times S) \cup$ $Z$. Clearly $Y$ is dense in $H$, as $F[[0, \lambda) \times \alpha S \subseteq Y \subseteq H$. We will show that $H=\beta Y$ by showing that $Y$ is $C^{*}$-embedded in $H$. We do this in two steps.

First, we show that $H \backslash\{a\}$ is $C^{*}$-embedded in $H$. To see this, observe that by $[\mathrm{PW}, 4 \mathrm{AG}(8)], \beta([0, \lambda) \times \alpha S=K$, and it quickly follows that $K=$ $\beta(K \backslash\{(\lambda, \lambda)\})$. Now $F^{\leftarrow}[F((\lambda, \lambda))]=\{(\lambda, \lambda)\}$, so $F \mid K \backslash\{(\lambda, \lambda)\}$ is a perfect continuous surjection from the almost compact space $K \backslash\{(\lambda, \lambda)\}$ onto $H \backslash\{a\}$. It follows that $H \backslash\{a\}$ is almost compact and noncompact, and hence $H \backslash\{a\}$ is $C^{*}$-embedded in $H$. (See [GJ, 6J] for relevant information about almost compact spaces.)

Next we claim that $Y$ is $C^{*}$-embedded in $H \backslash\{a\}$. By [GJ, 6H] it suffices to show that $Y$ is $C^{*}$-embedded in $Y \cup\{(\lambda, s)\}$ for each $s \in S$. Let $g \in C^{*}(Y)$ and let $M$ be a compact $S$-neighborhood of $s$. Then $[0, \lambda) \times M$ is $C^{*}$ embedded in $[0, \lambda] \times M$ (by Glicksberg's theorem yet again), so $g \mid[0, \lambda) \times$ $M$ extends continuously to $g^{\#} \in C^{*}([0, \lambda] \times M)$. Define $g^{*}: Y \cup([0, \lambda] \times$ int $\left._{S} M\right) \rightarrow \mathbb{R}$ by $g^{*} \mid Y=g$ and $g^{*} \mid[0, \lambda] \times$ int $_{S} M=g^{\#} \mid[0, \lambda] \times$ int $_{S} M$. Then by $[\mathrm{GJ}, 1 \mathrm{~A}(\mathrm{a})], g^{*}$ is continuous, so $g^{*} \mid Y \cup\{(\lambda, s)\}$ is the required continuous extension of $g$.

As $Y$ is $C^{*}$-embedded in $H \backslash\{a\}$ and $H \backslash\{a\}$ is $C^{*}$-embedded in $H$, it 
follows that $\beta Y=H$. Thus $\beta Y \backslash Y=E \cong S \cup\{\lambda\}$. It remains to show that $Y$ is normal.

Observe that $K \backslash(\{\lambda\} \times(S \cup\{\lambda\}))=[P \backslash\{(\lambda, \lambda)\}] \cup[[0, \lambda) \times \alpha S]$; hence it is the union of two countably compact subspaces and thus is itself countably compact. Clearly, $F[K \backslash(\{\lambda\} \times(S \cup\{\lambda\}))]=Y$, so $Y$ is also countably compact.

Now we show that $Y$ is normal. Let $A$ and $B$ be disjoint closed subsets of $Y$, and recall that $Y=([0, \lambda) \times S) \cup Z$. As $Z$ is normal and almost compact by Theorem 3, either $A \cap Z$ or $B \cap Z$ is compact. Suppose $A \cap Z$ is. Then there are disjoint open sets $U$ and $V$ of $Y$ such that $A \cap Z \subseteq U$ and $B \subseteq V \subseteq X \backslash A$. Now $A$ is countably compact as $Y$ is, so $A \backslash U$ is a countably compact closed subset of $[0, \lambda) \times S$. If $\Pi_{S}:[0, \lambda) \times S \rightarrow S$ is the projection map, then $\Pi_{S}[A \backslash U]$ is countably compact and hence pseudocompact. Hence, $\mathrm{cl}_{S} \Pi_{S}[A \backslash U]$ is pseudocompact closed subset of $S$, and hence by hypothesis is compact. Using the local compactness of $S$, we can find a compact subset $F$ of $S$ such that $\operatorname{cl}_{S} \Pi_{S}[A \backslash U] \subseteq \operatorname{int}_{S} F \subseteq F$. Thus $A \backslash U \subseteq[0, \lambda) \times \operatorname{int}_{S} F \subseteq$ $[0, \lambda) \times F$, and as $t(F) \leq t(S)<\operatorname{cf}(\lambda)$, by Theorem $1,[0, \lambda) \times F$ is normal. It follows that there are disjoint open sets $R$ and $Q$ in $[0, \lambda) \times F$ such that $A \backslash U \subseteq R$ and $B \cap[[0, \lambda) \times F] \subseteq Q$. Thus $A \subseteq U \cup\left[R \cap\left[[0, \lambda) \times\right.\right.$ int $\left.\left._{S} F\right]\right]=$ $C$ and $B \subseteq V \cap\left[Q^{\prime} \cup(Y \backslash([0, \lambda) \times F))\right]=D$, where $Q^{\prime}$ is open in $Y$ and $Q^{\prime} \cap([0, \lambda) \times F)=Q$. Then $C$ and $D$ are open in $Y$ and are easily verified to be disjoint. Consequently $Y$ is normal and the proof is complete.

\section{Applications}

We began with a straightforward application of Theorem 4 .

Example 1. Let $S=\left\{\alpha<\omega_{1}: \alpha\right.$ is a successor ordinal $\}$, and let $\alpha S=\left[0, \omega_{1}\right]$. Then $a S \backslash S \cong\left[0, \omega_{1}\right]$ and $\operatorname{cf}\left(\omega_{1}\right)=\omega_{1}>t(S)$. As $S$ is discrete, its pseudocompact closed subsets are compact. Hence, the hypotheses of Theorem 4 are satisfied, and so there exists a normal space $Y$ such that $\beta Y \backslash Y \cong S \cup\left\{\omega_{1}\right\}$. From our construction of $\alpha S$ it is clear that $S \cup\left\{\omega_{1}\right\}$ is the "one-point Lindelöf extension" of the discrete space of cardinality $\omega_{1}$; hence this space is the Stone-Čech remainder of a normal space.

Example 2. Let $\mathbb{N}$ denote the discrete space of positive integers. As in [vD], $t$ is defined to be the least cardinal $\kappa$ for which there is a family $\left\{B_{\alpha}: \alpha<\kappa\right\} \subseteq[\mathbb{N}]^{\omega}$ such that $\left\{B_{\alpha}^{*}: \alpha<\kappa\right\}$ is a strictly decreasing chain in $\mathbb{N}^{*}$ whose intersection is nowhere dense in $\mathbb{N}^{*}$. As noted in [vD], $\aleph_{0}<\mathbf{t} \leq 2^{\aleph_{0}}$ and $\mathbf{t}$ is a regular cardinal. Let $D=\bigcap\left\{B_{\alpha}^{*}: \alpha<\kappa\right\}, B_{\mathbf{t}}=\varnothing, B_{-1}=\mathbb{N}$, and $\alpha \mathbb{N}=\mathbb{N} \cup[0, \mathbf{t}]$. A subset $U \subseteq \alpha \mathbb{N}$ is defined to be open in $\alpha \mathbb{N}$ if $\alpha \in[0, \mathbf{t}] \cap U$ implies there is some $\beta<\alpha$ and a finite subset $F \subset \mathbb{N}$ such that $(\beta, \alpha] \cup B_{\beta} \backslash\left(B_{\alpha} \cup F\right) \subseteq U$.

It is straightforward to verify (see [FR, Examples 1.1 and 1.5] that $\alpha \mathbb{N}$ is a compactification of $\mathbb{N}$ and $\alpha \mathbb{N} \backslash \mathbb{N}=[0, t]$. By Theorem 4, there is a normal space $M$ such that $\beta M \backslash M=\mathbb{N} \cup\{\mathbf{t}\}$. The countable space $\mathbb{N} \cup\{\mathbf{t}\}$ will provide an answer to the first question of Fleissner and Levy (quoted in $\S 1$ if $\mathbb{N} \cup\{\mathbf{t}\}$ is not sequential. To show $\mathbb{N} \cup\{\mathbf{t}\}$ is not sequential, it suffices to show that $\mathbb{N}$ is sequentially closed in $\mathbb{N} \cup\{\mathbf{t}\}$, i.e., no infinite subset of $\mathbb{N}$ converges to $\mathbf{t}$. Suppose $A$ is an infinite subset of $\mathbb{N}$. Since $D$ is nowhere dense, there is an infinite subset $B$ of $A$ such that $B^{*} \subseteq A^{*} \backslash D$. By the compactness of $\mathbb{N}^{*}$, it follows there is some $\alpha<\mathrm{t}$ such that $B^{*} \cap B_{\alpha}^{*}=\varnothing$. So $B \cap B_{\alpha}$ is a finite subset 
of $\mathbb{N}$ and $(\alpha, \mathbf{t}] \cup B_{\alpha} \backslash\left(B \cap B_{\alpha}\right)$ is an open set missing $B$. Hence, $A$ does not converge to $\mathbf{t}$.

Example 3. Let $S=(\mathbb{N} \times \mathbb{N}) \cup\{\omega(n): n \in \mathbb{N}\} ; U \subseteq S$ is defined to be open if $\omega(n) \in U$ implies there is some finite subset $F \subset \mathbb{N}$ such that $\{n\} \times(\mathbb{N} \backslash F) \subseteq U$. Thus, $S_{n}=(\{n\} \times \mathbb{N}) \cup\{\omega(n)\}$ is the one-point compactification of $\{n\} \times \mathbb{N}$, and $S$ is the sum of the $S_{n}$ 's. For a subset $F \subseteq \mathbb{N}$, let $S_{F}=\bigcup\left\{S_{n}: n \in F\right\}$. For $f_{\alpha} \in{ }^{\mathbb{N}}$, let $T_{\alpha}=\bigcup\left\{\{n\} \times\left[1, f_{\alpha}(n)\right]: n \in \mathbb{N}\right\}$ and $T_{\mathbf{d}}=S$.

Let us assume the existence of a scale, i.e., a cardinal $\mathbf{d}$ and a cofinal family $\mathscr{F}=\left\{f_{\alpha}: \alpha<\mathbf{d}\right\}$ of $\left({ }^{\mathbb{N}} \mathbb{N}, \leq^{*}\right)$ such that $\mathscr{F}$ is well ordered by $\leq^{*}$. (Recall $f \leq^{*} g$ if $\{n \in \mathbb{N}: f(n)>g(n)\}$ is finite.) It is known that Martin's Axiom implies the existence of a scale. As noted in [vD], d is a regular cardinal. Without loss of generality, we can assume that $f_{0}(n)>1$ for all $n \in \mathbb{N}$ and for each $\alpha \leq \mathbf{d}, T_{\alpha} \backslash\left(\bigcup_{\beta \in \alpha} T_{\beta} \cup S_{F}\right)$ is infinite for all finite $F \subset \mathbb{N}$. Let $U(\alpha, \beta, F)=\left(T_{\alpha} \backslash\left(T_{\beta} \cup S_{F}\right)\right) \cup(\beta, \alpha]$, where $\beta<\alpha \leq \mathbf{d}$ and $F \subseteq \mathbb{N}$.

Let $\alpha S=S \cup[0$, d] . A subset $U$ of $\alpha S$ is defined to be open if (1) $U \cap S$ is open in $S$; (2) $0 \in U$ implies for some finite subset $F \subset \mathbb{N}, T_{0} \backslash S_{F} \subseteq U$; and (3) $\alpha \in(0$, d] $\cap U$ implies for some $\beta<\alpha$ and finite subset $F \subset \mathbb{N}$, $U(\alpha, \beta, F) \subseteq U$. It is easy to verify that $\alpha S$ is a Hausdorff extension of $S$. To show $\alpha S$ is compact, let $\mathbb{U}$ be an open cover of $\alpha S$. Since $[0$, d] is compact, there are $0=\alpha_{1}<\alpha_{2}<\cdots<\alpha_{n}=\mathbf{d}$ and finite sets $F_{1}, \cdots, F_{n}$ such that $U\left(\alpha_{i+1}, \alpha_{i}, F_{i}\right) \subseteq$ some $U_{i} \in \mathbb{U}$. Let $F=F_{1} \cup \cdots \cup F_{n}$. Then $\alpha S \backslash S_{F} \subseteq U_{1} \cup \cdots \cup U_{n}$. Since $S_{F}$ is compact, it follows that $\alpha S$ is compact. Thus, $\alpha S$ is a compactification of $S$ such that $\alpha S \backslash S=[0, \mathrm{~d}]$. By Theorem 4 , there is a normal space $M$ such that $\beta M \backslash M=S \cup\{\mathbf{d}\}$. Note that $U$ is an open neighborhood of $\mathbf{d}$ iff there is a finite subset $F \subset \mathbb{N}$ such that $\{\omega(n): n \in$ $\mathbb{N} \backslash F\} \subseteq U$ and $U \cap S_{n}$ is open in $S_{n}$ for each $n$. In particular, $S \cup\{\mathbf{d}\}$ is homeomorphic to the Arens Space (described in [F]) which is sequential but not locally compact. This provides an answer (assuming the existence of a scale) to the second question posed by Fleissner and Levy.

\section{A RESUlt ON NORMAL ALMOST COMPACT SPACES}

Throughout this section $X$ will denote a locally compact realcompact noncompact space, $\alpha X$ will be compactification of $X$, and $f: \beta X \rightarrow \alpha X$ will extend the identity map on $X$ continuously.

Theorem 5. Assume that there exists $p \in \alpha X \backslash X$ such that:

(1) $\beta((\alpha X \backslash X) \backslash\{p\})=\alpha X \backslash X$.

(2) $f^{\leftarrow}(p)$ is nowhere dense in $\beta X \backslash X$.

Then

(a) $\beta(\alpha X \backslash\{p\})=\alpha X$.

(b) The following are equivalent:

(i) $\alpha X \backslash\{p\}$ is normal.

(ii) $(\alpha X \backslash X) \backslash\{p\}$ is normal and $\alpha X \backslash\{p\}$ is countably compact.

Proof. (a) Evidently $\beta X \backslash f^{\leftarrow}(p)$ is the union of the locally compact realcompact space $X$ and the dense subspace $\beta X \backslash f^{\leftarrow}(p)$ of $\beta X \backslash X$. Hence, by [PW, 5.11 (i)(2)] $\beta X \backslash f^{\leftarrow}(p)$ is pseudocompact. Hence $f\left[\beta X \backslash f^{\leftarrow}(p)\right]=\alpha X \backslash\{p\}$, henceforth denoted by $L$, is pseudocompact. 
Let $Z, S \in \mathbf{Z}(L)$ with $Z \cap S=\varnothing$. Then there exists $g \in C^{*}(L)$ with $0 \leq g \leq 1$ such that $g[Z]=\{0\}$ and $g[S]=\{1\}$. Let $A=\operatorname{cl}_{L} g^{\leftarrow}\left[\left[0, \frac{1}{3}\right)\right]$ and $B=\operatorname{cl}_{L} g^{\leftarrow}\left[\left(\frac{2}{3}, 1\right]\right]$. Then $A \supseteq Z$ and $B \supseteq S$, so if we can show that one of $A$ and $B$ must be compact, then one of $Z$ and $S$ will be compact. As $Z$ and $S$ were arbitrarily chosen disjoint zero sets of $L$, this would prove (a) (see [GJ, $6 \mathrm{~J}]$ ). Now $A \backslash X$ and $B \backslash X$ are completely separated closed subsets of the space $L \backslash X$, which by (1) is almost compact, so at least one of them-say $A \backslash X$ - is compact. We will prove that $A$ is compact.

There is an open set $G$ in $L$ containing $A \backslash X$ such that $\operatorname{cl}_{L} G$ is compact. Then $H=\operatorname{cl}_{L}\left(A \backslash \mathrm{cl}_{L} G\right)$ is a regular closed subset of the pseudocompact space $L$ and hence is pseudocompact (see [PW, 1Q(2),(4)]). But $H$ is contained in $A$ and disjoint from $A \backslash X$; so $H$ is a (closed) subset of the realcompact space $X$ and hence is realcompact. This shows that $H$ is compact. As $A \subseteq H \cup \operatorname{cl}_{L} G$, $A$ is compact and (a) follows.

(b) (i) $\Rightarrow$ (ii): If $\alpha X \backslash\{p\}$ is normal, so is its closed subspace $(\alpha X \backslash X) \backslash\{p\}$. By (a), $\alpha X \backslash\{p\}$ is pseudocompact and hence (as it is normal) it is countably compact.

(ii) $\Rightarrow$ (i): Let (ii) hold and let $A$ and $B$ be disjoint closed subsets of $\alpha X \backslash\{p\}$ (which, recall, we denote by $L$ ). We will show that at least one is compact; hence $L$ will be normal.

As $L \backslash X$ is normal and almost compact, at least one of $A \backslash X$ and $B \backslash X$ is compact; suppose $A \backslash X$ is. We show that $A$ is compact. There is an open set $G$ in $L$ containing $A \backslash X$ such that $\operatorname{cl}_{L} G$ is compact. The closed subset $A \backslash G$ of $L$ is countably compact. Also, $A \backslash G$ is a closed subset of $X$ (which is realcompact); so $A \backslash G$ is compact. As $A \subseteq(A \backslash G) \cup \operatorname{cl}_{L} G, A$ is compact.

\section{ACKNOWLEDGMENT}

The authors would like to thank the referee for several useful suggestions, in particular, the inclusion of Example 1 of $\S 3$.

\section{REFERENCES}

[vD] E. K. van Douwen, The integers and topology, Handbook of Set-Theoretic Topology (K. Kunen and J. Vaughan, eds.), North-Holland, Amsterdam, 1984, pp. 111-167.

[F] S. P. Franklin, Spaces in which sequences suffice. II, Fund. Math. 61 (1967), 51-56.

[FL] W. Fleissner and R. Levy, Stone-Čech remainders which make continuous images normal, Proc. Amer. Math. Soc. 106 (1989), 839-842.

[FR] S. P. Franklin and M. Rajagopalan, Some examples in topology, Trans. Amer. Math. Soc. 155 (1971), 305-314.

[GJ] L. Gillman and M. Jerison, Rings of continuous functions, Van Nostrand, Princeton, NJ, 1960.

[H] R. Hodel, Cardinal functions. I, Handbook of Set-Theoretic Topology (K. Kunen and J. Vaughan, eds.), North-Holland, Amsterdam, 1984, pp. 1-61.

[M] K. Magill, A note on compactifications, Math. Z. 94 (1966), 322-325.

[N] T. Nogura, Tightness of comapct Hausdorff spaces and normality of product spaces, J. Math. Soc. Japan 28 (1976), 360-362.

[P] T. Przymusinski, Products of normal spaces, Handbook of Set-Theoretic Topology (K. Kunen and J. Vaughan, eds.), North-Holland, Amsterdam, 1984, pp. 781-826. 
[PW] J. R. Porter and R. G. Woods, Extensions and absolutes of Hausdorff spaces, Springer-Verlag, New York, 1987.

[V] J. Vermeer, Two generalizations of normality, Topology and Measure III, Proc. Conf. (Vitte/Hiddensee, 1980), Part 2, 1982, pp. 329-338.

Department of Mathematics, University of Kansas, LAWrence, KaNSAS 66045

Department of Mathematics, University of Manitoba, Winnipeg, Manitoba, Canada R3T 2N2 